\title{
Kompressionstherapie
}

\section{Gut gewickelt?}

\author{
Die Kompressionstherapie bildet eine Säule bei der Behandlung \\ des Ulcus cruris venosum. Dennoch werden viele Patienten nicht \\ optimal versorgt. Gibt es Möglichkeiten zur Verbesserung \\ der Patientenversorgung?
}

? Frau Protz, wie ist es um die Kompressionstherapie in Deutschland bestellt?

Protz: Es könnte besser sein. Versorgungsstudien belegen, dass bis zu $60 \%$ der Patienten mit einem UCV keine Kompressionstherapie erhalten. Diese komplexe Therapieform erfordert praktische Fertigkeiten, pathophysiologische Kenntnisse, einen Überblick über die aktuell verfügbaren Materialien und die in Frage kommenden Methoden. In all diesen Bereichen haben die Anwender Probleme und Defizite. In einem deutschlandweit durchgeführten Praxistest gelang es lediglich 51 von 551 Teilnehmern mit einer Kurzzugbindenbandagierung nach der modifizierten Sigg-Technik, einen therapierelevanten Druckwert im Bereich 50$60 \mathrm{mmHg}$ zu erzeugen. Zudem zeigte eine aktuelle Patientenbefragung, dass viele Betroffene nicht ausreichend versorgt und informiert sind. So kannten sie sich weder optimal mit der Pflege ihrer Binden oder medizinischen Kompressionsstrümpfe noch mit einer adäquaten Hautpflege aus.

? Woran liegt das und wie lässt sich das ändern?

Protz: Während der Pflegeausbildung wird die praktische Durchführung der Kompressionstherapie meist nur in ein bis zwei Unterrichtsstunden vermittelt, im Medizinstudium nahezu gar nicht. Hier wäre eine entsprechende Anpassung der Ausbildungsinhalte sinnvoll. Auf Versorgerseite lässt sich die Ausführung von Kompressionsbandagierungen durch ständiges Üben sicherlich deutlich verbessern. Spezielle Messsonden, die den Druck un- terhalb der Bandagierung messen, helfen, den therapierelevanten Druck zu erreichen. Aufgrund der hohen Anschaffungskosten sind diese Geräte jedoch nicht überall verfügbar. Solche praktischen Übungen müssen regelmäßig durch Schulungen und Fortbildungen ergänzt werden. Versorger sollten nicht nur mit der Anwendung der Materialien der Kompressionstherapie vertraut sein, sondern auch mit der Vielzahl der Produkte. Für eine leitliniengerechte, sach- und fachgerechte Versorgung, die sich an den Patientenbedürfnissen und -fähigkeiten orientiert, wären spezialisierte Versorger wünschenswert.

? Die Kompressionstherapie steht und fällt mit der Adhärenz der Patienten. Von welchen Faktoren hängt diese ab? Protz: Viele Betroffene bringen eine lange Krankengeschichte mit vielen Vorversorgern mit sich. Neuen Versorgern und Methoden begegnen sie häufig mit Misstrauen. Aber auch das Festhalten an einer bestimmten Versorgungsform kann dem nächsten Therapieschritt im Wege stehen. Die Patienten beharren dann auf ihrem alten Strumpf, der bequem sitzt, aber keinen therapierelevanten Druck leistet.

Generell sollte die Therapie für die Patienten so effektiv und angenehm wie möglich sein. So schützt die Verwendung von Polstermaterialien die Haut unterhalb der Kompressionsbandagierung und beugt Schmerzen durch einschnürende Bindentouren vor. Dadurch wird der erforderliche Anlagedruck für den Patienten erträglicher und die Adhärenz gesteigert. Nach drei bis vier Wochen sollte die initiale Entstauungsphase abge-

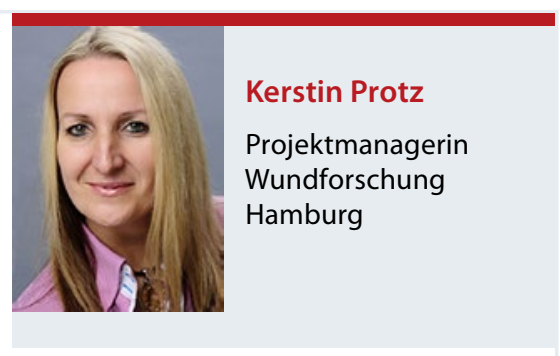

schlossen sein und die Versorgung auf eine Bestrumpfung, z.B. in Form von UIcus-Strumpfsystemen, umgestellt werden. Strümpfe sind einfacher und oft auch selbstständig anzulegen. Zudem schränken sie die Kleider- und Schuhauswahl nicht so ein wie dicke Wickelungen.

? Wie können Pflegende die Patienten besser unterstützen?

Protz: Die Mitwirkung des Patienten ist entscheidend für den Erfolg der Kompressionstherapie. Sie sollte motivierend unterstützt werden. Es geht um ein aktives Einbringen in den Versorgungsprozess. Unabhängigkeit von Anderen ist für die meisten Menschen eine Basis für Zufriedenheit. Individuell angepasste An- und Ausziehhilfen erleichtern den selbstständigen Umgang mit Kompressionsstrümpfen. Patienten sollten aber auch über die Vielfalt des Angebots, modische Varianten und Neuheiten informiert werden. So können adaptive Kompressionsbandagen, auch Wrap- oder Klettverbände genannt (z.B. JuxtaCures ${ }^{\circledast}$ ), die Akzeptanz für eine Kompressionstherapie erhöhen. Solche Bandagen sind durch Klett-Verschlüsse relativ einfach selbst anzulegen.

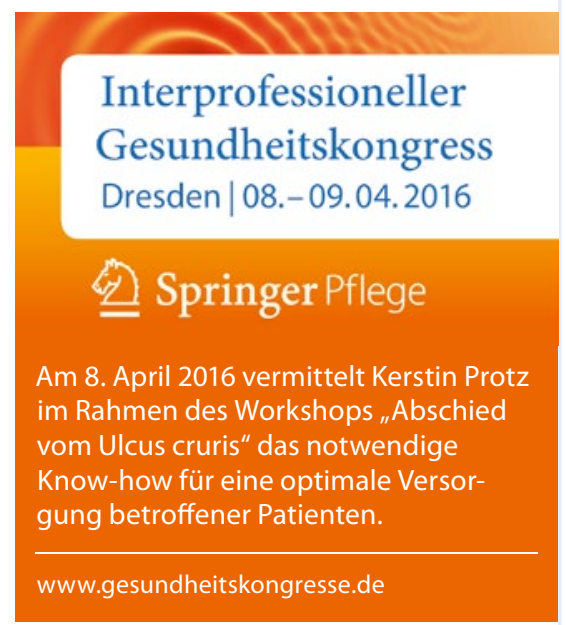

